\title{
Hollow in the Lung with Hoarseness: An Uncommon Association with Pulmonary Thromboembolism
}

\section{Sushil Upadhyay*}

Rama Medical College and Hospital, Ghaziabad, Uttar Pradesh, India

\begin{abstract}
A case is presented where focus of all investigations centred around a lung cavity which in association hoarseness appeared in all probability a lung cancer after no response to anti tuberculosis chemotherapy. Oxygen desaturation on few steps of walk lead to further work up concluding with diagnosis of pulmonary thromboembolism with cavitary infract and cardiovocal syndrome.
\end{abstract}

Keywords: Cavitary infarct; Pulmonary thromboembolism; Cardiovocal syndrome

\section{Introduction}

Pulmonary infarction is an infrequent complication of pulmonary thromboembolism owing to dual blood supply and rich capillary anastomosis. Liquefaction of pulmonary infarct-cavitary infarct is an unusual phenomenon. We report a case of cavitary lung disease in a 53 year old previously healthy non-smoker male who was treated for pulmonary tuberculosis without clinical improvement and ultimately proved to be a case of pulmonary thromboembolism with cavitary infarct. Hoarseness, another remarkable symptom in this case is also an exceptionally rare association with secondary pulmonary hypertension.

\section{Case Report}

A 53 year old male presented with non-productive cough and breathlessness on exertion. The internist referred the patient to pulmonology for bronchoscopy. Patient had a chest X-ray and CT scan with large left upper lobe cavity and he had not responded to four months of anti-tuberculosis treatment. Pulmonary malignancy was considered as other possible diagnosis.

Prior to visiting us, patient had been through number of physician and specialty consultations for almost 10 months.

Patient had been in good health till recent past. He was a never smoker and tea-totalar with atheletic built. His problems begin with shortness of breath, which he appreciated during morning walks. Few weeks later, he developed common cold and cough, when he showed to a physician. Routine blood test picked up eosinophilia. Chest X-ray (Figure 1) was reported unremarkable. Spirometry (Figure 2) showed mild obstructive ventilator defect. He was prescribed diethyl carbazine (DEC), inhaled bronchodilators with steroid and antihistamines. Patient got no remarkable relief in symptoms. Cough persisted and shortness of breath progressed to the extent that he discontinued his morning walks.

He presented to a cardiologist. Stress test is negative for reversible ischemia. Echocardigram is reported to have mild RA and RV dilatation with normal LV function. Repeat chest skiagram and CT scan (Figures 3 and 4) now show left parahilar consolidation with breakdown. He is prescribed anti TB treatment after consultation with a chest physician.

Patient continued anti tuberculosis drugs, but even after four months of treatment, he continued to be symptomatic. At this time he developed hoarseness, for which he was referred to ear nose throat (ENT) specialist. Laryngeal examination showed fixed left vocal cord. Repeat Chest X-ray and CT scan (Figures 5 and 6) revealed a large left upper lobe cavity. Patient presents to us with this background of

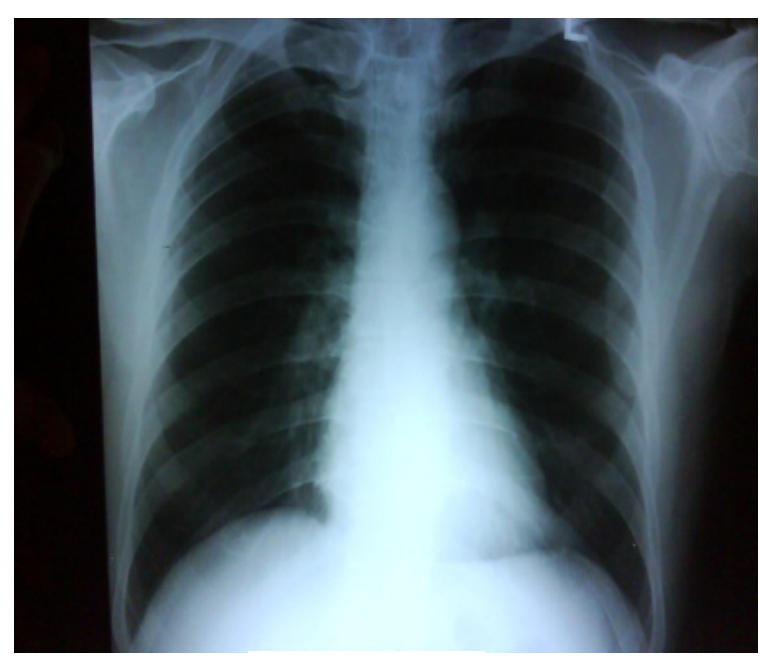

Figure 1: CXR: May 2008.

information and records.

Patient is admitted for further workup. Physical examination is remarkable with hoarse voice, resting tachypnea (RR:22 BPM) and tachycardia (PR:132/Minute, Regular and good volume). Chest and precordial auscultation reveal normal breath sounds and increased heart rate respectively. Oxygen saturation $(\mathrm{spO} 2)$ on ambient air is $94 \%$. Other systemic examination is normal. Patient is made to walk in the corridor. Marked shortness of breath (Borge scale 8/10) along with desaturation (spO2 74\%) and tachycardia (PR 162/Minute) is noted during few meters of walk.

Findings of exercise desaturation lead to suspect thromboembolic phenomenon and investigations directed to pulmonary thromboembolism (PTE) are perused. 2D echocardigram reveals markedly dilated RA and RV, diminished RV contractility and

${ }^{*}$ Corresponding author: Sushil Upadhyay, MD (Chest), Assistant Professor Rama Medical College and Hospital, Ghaziabad, Uttar Pradesh, India, Tel: +919899393006; E-mail: skupadhyay2007@gmail.com

Received March 11, 2016; Accepted April 14, 2016; Published April 18, 2016

Citation: Upadhyay S (2016) Hollow in the Lung with Hoarseness: An Uncommon Association with Pulmonary Thromboembolism. J Pulm Respir Med 6: 334 doi:10.4172/2161-105X.1000334

Copyright: (c) 2016 Upadhyay S. This is an open-access article distributed under the terms of the Creative Commons Attribution License, which permits unrestricted use, distribution, and reproduction in any medium, provided the original author and source are credited. 


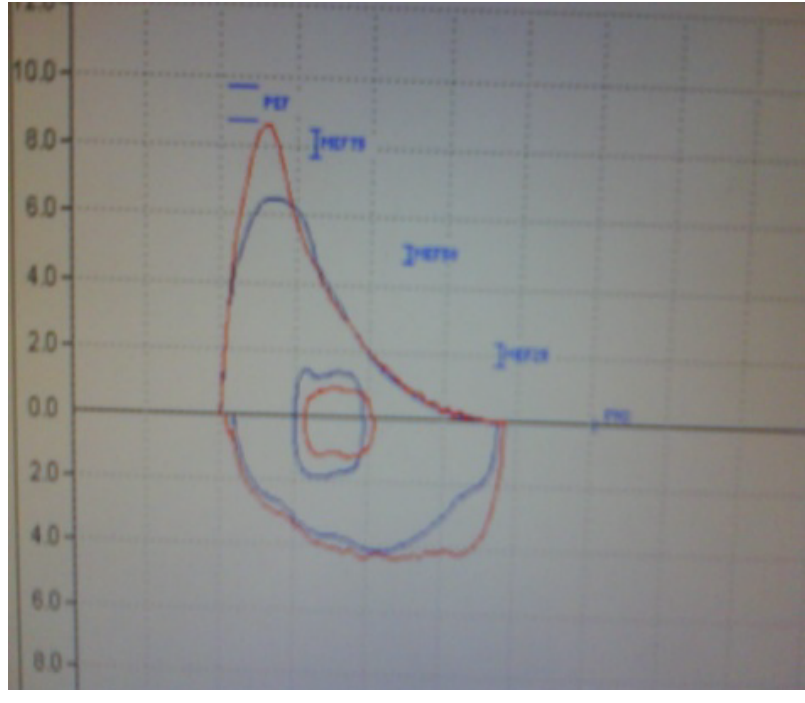

Figure 2: Spirometry: Mild obstruction.

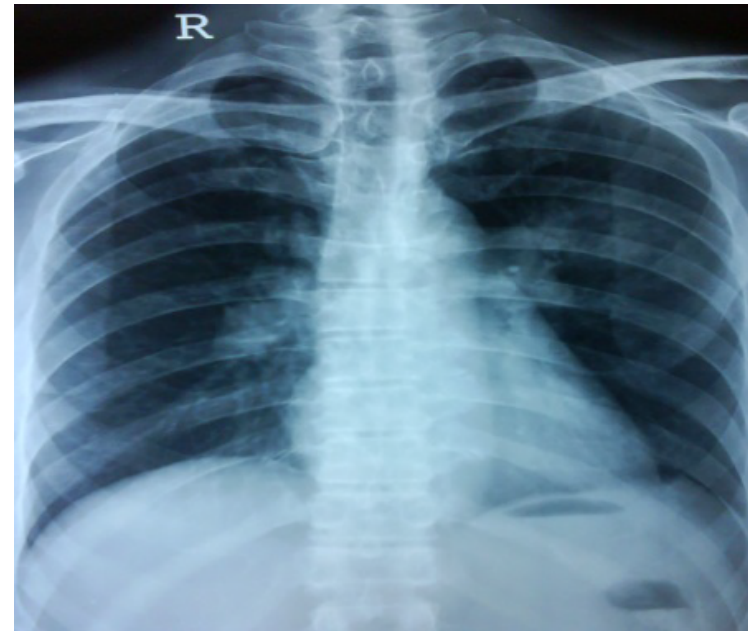

Figure 3: CXR: Aug 2008.

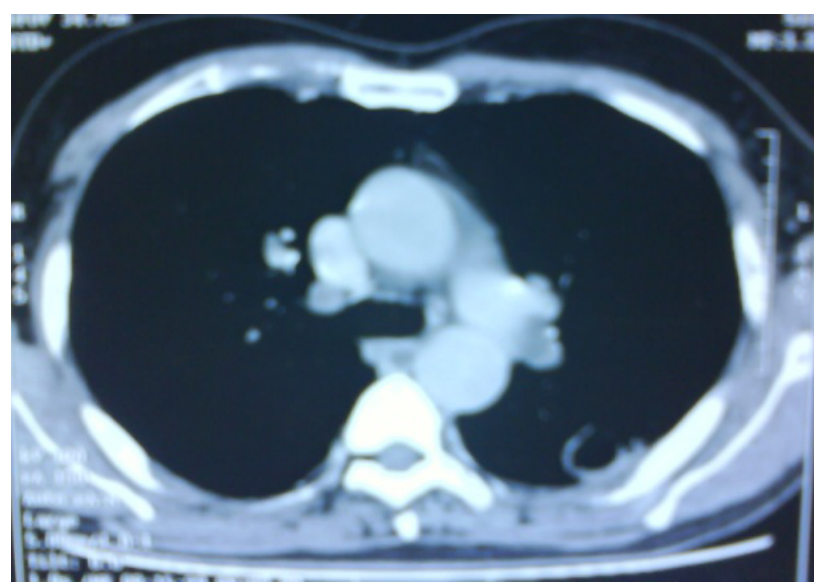

Figure 4: CT thorax: Aug 2008.

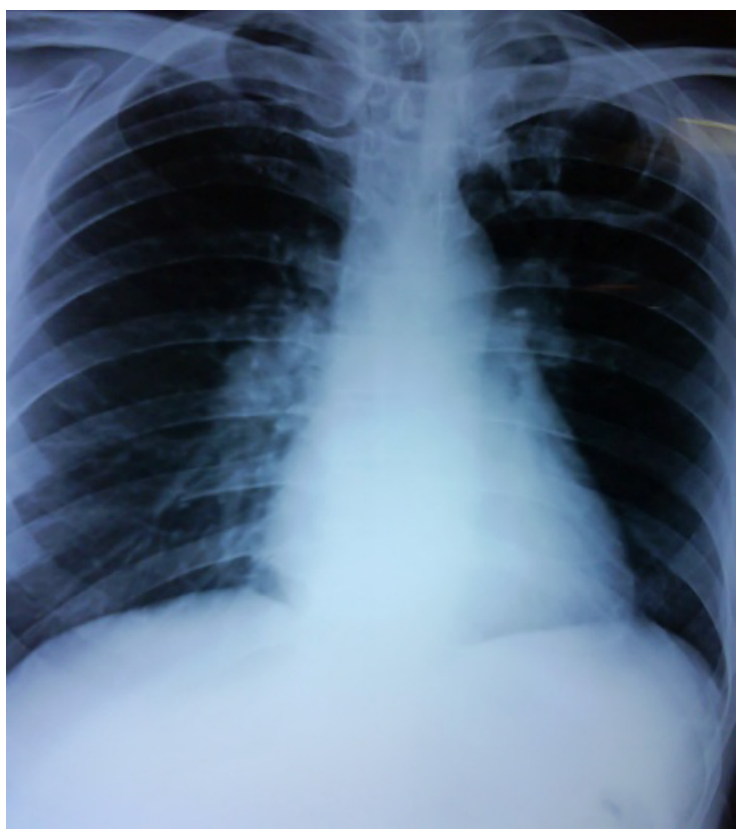

Figure 5: CXR: Nov 2008
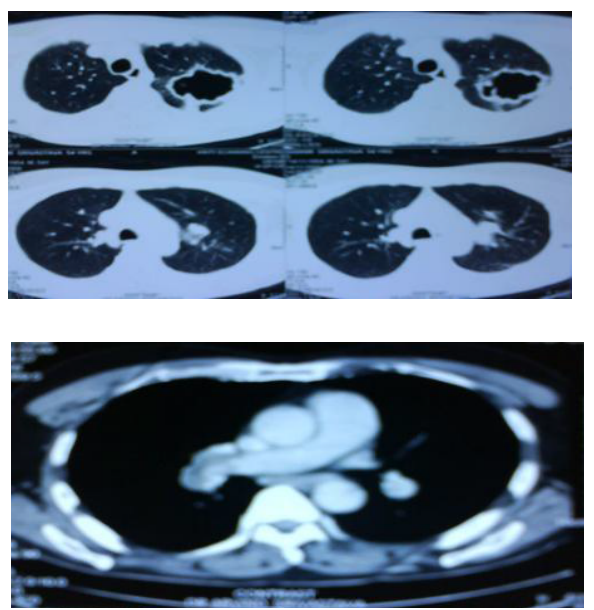

Figure 6: CT thorax: LUL cavity.
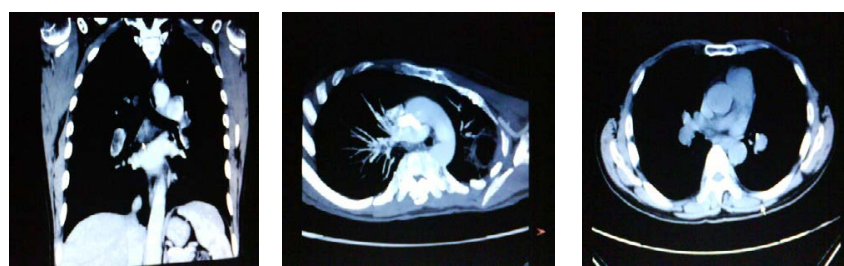

Figure 7: CT pulmonary angiogram: Filling defects in descending pulmonary arteries and LUL cavity.

pulmonary artery systolic pressure (PASP) of $>60 \mathrm{mmHg}$. CT pulmonary angiogram (Figure 7) shows shaggy walled cavitating lesion in left upper lobe measuring $7.3 \times 6.1 \times 5.0 \mathrm{~cm}$ with speculated margins laterally, posteriorly, supero-medially reaching up to pleural surface. Multiple filling defects are noted in descending branches of bilateral 


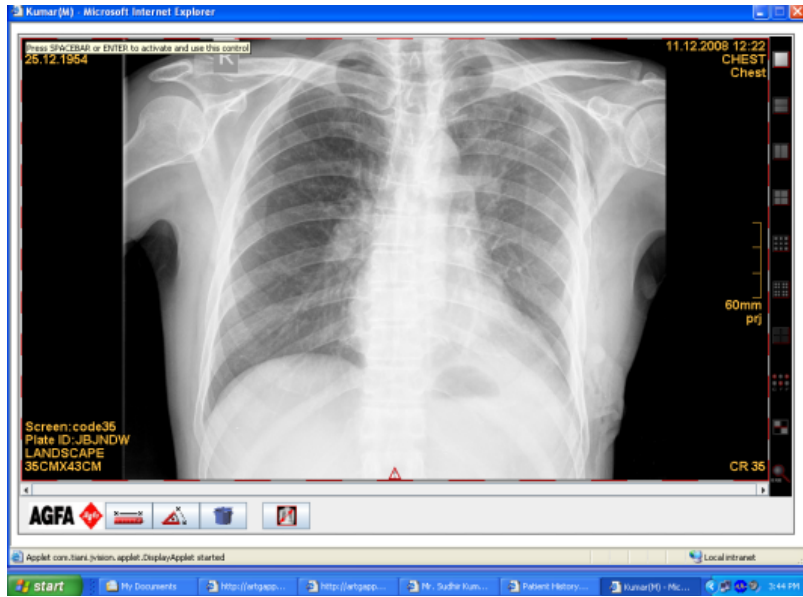

Figure 8: CXR: Post LUL wedge resection.

pulmonary arteries and upper lobe branch of RPA. Venous Doppler lower limbs picks up chronic deep vein thrombosis in distal half of right superficial femoral vein. Laboratory investigations of patient are remarkable for eosinophilia which was related to background history of allergic rhinitis. Thrombophilia profile was remarkable for Protein $\mathrm{C}$ deficiency. Bronchoscopy is done as requested by the admitting physician and it was normal except for left vocal cord palsy (Figure 8 ). Bronchial washings are negative for malignancy in cytology and negative for $\mathrm{AFB}$ on $\mathrm{ZN}$ staining.

Patient is started on low molecular weight heparin. He responds with remarkable relief in symptoms. There is remarkable decrease in pulmonary artery pressure on review echocardiogram. Patient is continued on low molecular weight heparin. However, the cause of hoarseness still remains unexplained. He is prepared for thoracotomy to rule out any possibility of malignancy. Left upper lobe wedge resection is done (Figure 9). Histopathology reveals extensive inflammation and fibrosis with organized thrombi in supplying vessels. IVC filter is placed in the femoral vein in the same sitting. Patient is discharged in improved general condition on oral anticoagulant and follows regularly as out patient. International Normalized Ratio (INR) is maintained between $2-3$. His hoarseness completely recovered in 2 months and he is able to continue his professional career and active personal life as before.

\section{Discussion}

Cavitatory lung disease can be caused by a wide variety of pathologic conditions. Possible etilogies include infection, metastatic malignancies, septic pulmonary emboli, granulomatous vasculitides and rarely pneumoconiosis and pulmonary sequestration. Cavitation resulting from bland pulmonary infarction is often not considered in differential diagnosis [1-4]. Pulmonary infarction occurs in only $10 \%$ of patients of pulmonary thromboembolism. Cavitation after pulmonary infarction is even a rare event. Large autopsy series reveal cavitation in $4-5 \%$ of all pulmonary infracts [3]. It is general agreement that cavitation occurs when the infarct is more than $4 \mathrm{cms}$ in size. It chiefly involves the upper and middle zones of the lungs with only $20 \%$ involvement of the lower lobes. Morphologically, the infarct is typically haemorrhagic with coagulative necrosis of parenchymal frame work, which heals with minimal fibrosis. On the other hand liquefactive necrosis is unusual with incidence of $2-4 \%$. Liquefaction usually follows septic thromboembolism, though it may occur in bland thrombi $[3,5,6]$. The natural history of cavitary infarctions is not well documented
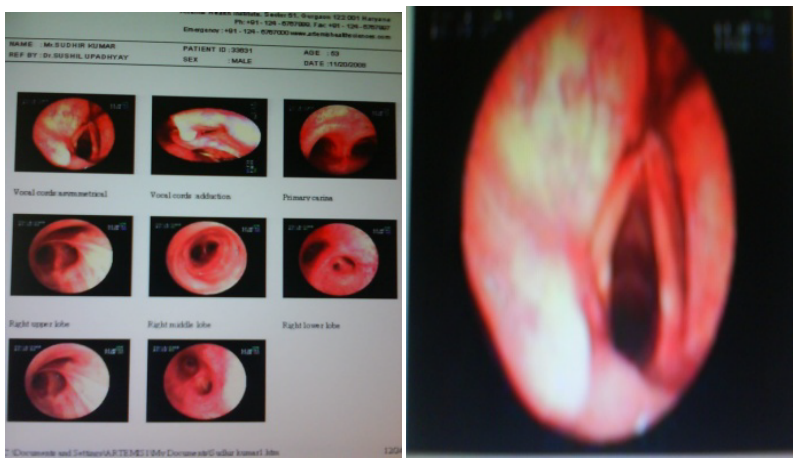

Figure 9: Video laryngobronchoscopy: LVC palsy.

or understood. There have been number of case reports describing various complications and high mortality rates associated with this condition. Mortality rate as high as $41 \%$ in non-infected and $73 \%$ in infected pulmonary infarcts have been reported. An aggressive surgical approach to management of cavitary infarcts with use of measures to prevent further emboli has been advocated by some authors. It is important to remember that cavitary pulmonary infarction though rare but nevertheless forms one of the differential diagnosis of cavitary lung lesions $[7,8]$.

Hoarseness was a prominent complaint in our patient and videolaryngoscopy confirmed the left vocal cord palsy. Ortner's Syndrome (described 118 years ago in 1897) is a clinical entity with hoarseness due to a left recurrent laryngeal nerve (LRLN) palsy caused by cardiac disease [9]. Left recurrent laryngeal nerve palsy has been reported with extreme rarity in association with moderate to severe pulmonary hypertension [10]. The mechanical cause has been advocated due to compression of RLN between enlarged pulmonary artery and aorta at ligamentum arteriosum [11].

\section{Conclusion}

This case is unique because of following reasons:

1. The difficulty in diagnosis imposed by conundrum of breathlessness and hoarseness with angry looking large cavity in left upper lobe lung. Prima-facie considered as tuberculosis or malignancy but ultimately turned out to be venous thromboembolism with cavitary infarct. This naturally incurred hot debates among the treating team viz. physician, pulmonolist, radiologists and cardiovascular surgeon.

2. The hoarseness, which confused and misdirected the attention towards malignancy and even lead to lung surgery later, could get explanation of cardiovocal syndrome (Ortner's syndrome).

3. Last but not the least, the simple observation which gave the clue towards embolic phenomenon was not anything special but the digital pulse oxymetry during walk picking up exercise desaturation.

\section{References}

1. Ryu JH, Swensen SJ (2003) Cystic and Cavitary Lung diseases: focal and diffuse. Mayo Clin Proc 78: 744-752.

2. Sainz A, Navarro L (2006) Cavitaion Pulmonary Non-infectious in an immunocompromised Host. The Internet J Pulmonary Med 6: 2.

3. Wilson AG, Joseph AE, Butland RJ (1986) The radiology of aseptic cavitation in pulmonary infarction. Clin Radiol 37: 327-333.

4. Libby LS, King TE, Laforce FM, Schwarz MI (1985) Pulmonary cavitation following pulmonary infarction. Medicine (Baltimore) 64 : 342-348. 
Citation: Upadhyay S (2016) Hollow in the Lung with Hoarseness: An Uncommon Association with Pulmonary Thromboembolism. J Pulm Respir Med 6: 334. doi:10.4172/2161-105X.1000334

Page 4 of 4

5. Aslam PA, Eastridge CE, Hughes FA (1970) Pulmonary cavitation after embolism. Int Surg 53: 157-161.

6. Bao YQ, Jin LR, Chen WC (1990) Pulmonary infarction presenting as aseptic cavitation. Chin Med J (Engl) 103: 689-691.

7. May IA, Samson PC, Mittal A (1972) Surgical management of the patient with complications of pulmonary infarction due to nonseptic pulmonary emboli. Am J Surg 124: 223-238.

8. Vidal E, LeVeen HH, Yarnoz M, Piccone VA Jr (1971) Lung abscess secondary to pulmonary infarction. Ann Thorac Surg 11: 557-564.
9. Thirlwall AS (1977) Ortner's syndrome: a centenary review of unilatera recurrent laryngeal nerve palsy secondary to cardiothoracic disease. J Laryngol Otol 11: 869-871.

10. Nakao M, Sawayama T, Samukawa M, Mitake H, Nezuo S, et al. (1986) Left recurrent laryngeal nerve palsy associated with primary pulmonary hypertension and patent ductus arteriosus. J Am Coll Cardiol 5: 788-792.

11. Zamora Mestre S, Ladrón de Guevara Bravo F, Acosta Varo M (1997) Paralysis of the left recurrent laryngeal nerve secondary to periprosthetic mitral insufficiency. Rev Esp Cardiol 50: 902-903. 